J. Lake Sci. (湖泊科学), 2019, 31(5): 1357-1367

DOI 10. 18307/2019. 0525

(c) 2019 by Journal of Lake Sciences

\title{
抚仙湖有色可溶性有机物的来源组成与时空变化
}

\author{
陈 乐 $^{1,2}$, 周永强 ${ }^{3}$, 周起超 ${ }^{2,4 * *}$, 李凯迪 ${ }^{2,5}$, 张运林 $^{3}$, 赵玉伟 ${ }^{6}$, 陆轶峰 ${ }^{5}$, 常军军 ${ }^{* *}$ \\ (1:云南大学国际河流与生态安全研究院,昆明 650500) \\ (2:云南省环境科学研究院云南省高原湖泊流域污染过程与管理重点实验室,昆明 650034) \\ (3: 中国科学院南京地理与湖泊研究所湖泊与环境国家重点实验室,南京 210008) \\ (4:云南大学资源环境与地球科学学院/高原湖泊生态与治理研究院,昆明 650500) \\ (5:云南大学生态学与环境学院,昆明 650500) \\ (6:玉溪市抚仙湖管理局,玉溪 653100)
}

\begin{abstract}
摘 要: 基于 2017 年 1-12 月在抚仙湖开展的逐月观测,利用紫外一可见吸收光谱和三维苂光光谱技术探讨该湖有色可溶 性有机物 (CDOM) 的来源组成及时空变化特征. 12 个月 CDOM 吸收值 $a(254)$ 的均值为 $3.47 \pm 0.57 \mathrm{~m}^{-1}$, 范围为 $1.82 \sim 5.22$ $\mathrm{m}^{-1}$, 说明 CDOM 丰度较低. 平行因子分析结果给出了 2 种类酪氨酸苂光组分 (C1 和 C3)、1 种类色氨酸苂光组分 (C2)、1 种 类腐殖质苂光组分 $(C 4), 12$ 个月内源组分 $(\mathrm{C} 1+\mathrm{C} 3)$ 对总苂光强度的平均贡献为 $65.81 \% \pm 15.38 \%$,外源组分 $(\mathrm{C} 2+\mathrm{C} 4)$ 的平均 贡献为 $34.19 \% \pm 15.38 \%$; 苂光指数 $F I$ 的均值为 $1.73 \pm 0.14$, 腐殖化指数 $H I X$ 的均值为 $1.02 \pm 0.37$, 生源化指数 $B I X$ 的均值为 $1.23 \pm 0.27$, 说明 $\mathrm{CDOM}$ 主要为微生物内源产生. 时空变化方面, 春 $(3-5$ 月)、夏 $(6-8$ 月)、秋( $9-11$ 月) 和冬 ( 1 、2、12 月) 季 的 $a(254)$ 分别为 $3.20 \pm 0.47 、 3.76 \pm 0.64 、 3.67 \pm 0.50$ 和 $3.23 \pm 0.38 \mathrm{~m}^{-1}$, 夏季和秋季均显著高于冬季和春季; CDOM 丰度及内外 源组分的空间分布具有季节异质性,可能与流域土地利用、河流输人、降雨、温度、光辐射等因素有关.
\end{abstract}

关键词: 有色可溶性有机物; 紫外一可见吸收光谱;三维若光光谱;平行因子分析;云南高原;抗仙湖

\section{Sources, composition and spatiotemporal variations of chromophoric dissolved organic matter in a deep oligotrophic Lake Fuxian, China}

CHEN Le ${ }^{1,2}$, ZHOU Yongqiang ${ }^{3}$, ZHOU Qichao ${ }^{2,4 * *}$, LI Kaidi ${ }^{2,5}$, ZHANG Yunlin ${ }^{3}$, ZHAO Yuwei ${ }^{6}$, LU Yifeng $^{5}$ \& CHANG Junjun ${ }^{\text {*** }}$

(1: Institute of International River and Eco-security, Yunnan University, Kunming 650500, P.R. China)

(2: Yunnan Key Laboratory of Pollution Process and Management of Plateau Lake-Watershed, Yunnan Institute of Environmental Science, Kunming 650034, P.R. China)

(3: State Key Laboratory of Lake Science and Environment, Nanjing Institute of Geography and Limnology, Chinese Academy of Sciences, Nanjing 210008, P.R. China)

(4: Institute for Ecological Research and Pollution Control of Plateau Lakes, School of Resource Environment and Earth Science, Yunnan University, Kunming 650500, P.R. China)

(5: School of Ecology and Environmental Science, Yunnan University, Kunming 650500, P.R.China)

(6: Yuxi Fuxian Lake Administration, Yuxi 653100, P.R.China)

Abstract: Lakes are important in terrestrial carbon cycling. Source and optical composition of chromophoric dissolved organic matter ( CDOM) in oligotrophic and deep lakes can display distinct properties, because of deep light penetration and long water residence time in these lakes. In this study, the optical properties and spatiotemporal distributions of CDOM were analyzed through monthly field investigation in 2017 in Lake Fuxian, an oligotrophic deep lake in Yunnan Province, China. The results showed that

* 云南省科技计划项目 (2016RA081，2017FD029)、国家自然科学基金项目 (41601208，41621002) 和云南省环境科 学研究院创新团队计划项目联合资助. 2018-12-10 收稿; 2019-04-02 收修改稿. 陈乐 (1994 ), 男, 硕士研究 生; E-mail: 18608839017@163.com.

** 通信作者;E-mail: qchzhou@ynu.edu.cn, changjunjun@ynu.edu.cn. 
the average value of $a(254)$ was $3.47 \pm 0.57 \mathrm{~m}^{-1}$, with the range of $1.82-5.22 \mathrm{~m}^{-1}$, indicating that CDOM abundance in the lake was relatively low compared with other mesotrophic and eutrophic lakes. Moreover, parallel factor analysis was performed to assess CDOM composition from excitation-emission matrix spectra and four components were identified: two tyrosine-like components (C1 and C3), one tryptophan-like component ( C2 ) and one humic-like component ( C4). The percentage of fluorescent intensity of $\mathrm{C} 1+\mathrm{C} 3$ was $65.81 \% \pm 15.38 \%$, and the proportion of $\mathrm{C} 2+\mathrm{C} 4$ was $34.19 \% \pm 15.38 \%$. The fluorescence index $(F I)$, humification in$\operatorname{dex}(H I X)$ and biological/autochthonous index $(B I X)$ was $1.73 \pm 0.14,1.02 \pm 0.37$ and $1.23 \pm 0.27$, respectively. These results demonstrated that the CDOM was primarily originated from endogenous microbes in this lake. The average values of $a(254)$ in spring (March-May), summer (June-August), autumn (September-November) and winter (January, February and December) were $3.20 \pm 0.47,3.76 \pm 0.64,3.67 \pm 0.50$ and $3.23 \pm 0.38 \mathrm{~m}^{-1}$ respectively, with significantly higher values in summer and autumn than those in winter and spring. The abundance and spatial distributions of autochthonous and allochthonous CDOM exhibited seasonal heterogeneity, which might be correlated with land-use pattern, input of terrestrial materials, rainfall, water temperature and irradiance.

Keywords: Chromophoric dissolved organic matter (CDOM); UV-visible spectroscopy; fluorescence spectroscopy; parallel factor analysis (PARAFAC); Yunnan Plateau; Lake Fuxian

天然水体中溶解性有机物 (dissolved organic matter, DOM) 是地球上最大的有机碳库, DOM 的来源主要 包括陆源的输人 (外源) 和微生物、藻类等的释放 (内源 ${ }^{[1-2]}$, 其迁移转化过程与碳循环及气候变化过程息 息相关 ${ }^{[3-4]}$. 湖泊接纳上游水系携人的陆源 DOM, 经一系列合成及微生物与光化学降解过程, 最后难降解的 DOM 继续向下游输移, 因而湖泊是地表碳循环的重要枢纽 ${ }^{[5-6]}$. 光化学降解与湖泊太阳辐射尤其是紫外短 波辐射的漫衰减息息相关, 而微生物降解程度可随湖泊水力滞留时间的延长而显著上 升 $^{[7]}$, 意味着清澈型 深水湖泊 DOM 的转化特征或不同于其他类型的湖泊. 气象 ${ }^{[2,8]}$ 、水文 ${ }^{[9]}$ 等自然条件与人类活动 ${ }^{[10]}$ 的变化可 对湖泊 DOM 的来源产生重要影响, 而水体 DOM 组成的微小变化可引起水生态系统的巨大变化 ${ }^{[11]}$. 例如, 陆源 DOM 的输人增加可导致水体二氧化碳及甲烷排放量增加 ${ }^{[12]}$ 、初级生产量减少 ${ }^{[13-14]}$, 故湖泊生态系统 中 DOM 的来源组成与含量的变化值得关注.

DOM 由腐殖酸、富里酸、脂肪族及芳烃聚合物等一系列结构复杂的有机物构成, 运用传统的化学分析手 段难以准确揭示其浓度及组成结构的变化情况 ${ }^{[15]}$. 值得指出的是有色可溶性有机物 ( chromophoric dissolved organic matter, CDOM) 是 DOM 中能强烈吸收紫外辐射的那部分物质, 因而运用 CDOM 吸收光谱特征波段及 波谱形态特征即可在一定程度上揭示 DOM 浓度与组成 ${ }^{[16]}$. 另外还有一部分 DOM 在短波激发条件下会发 出苂光, 这部分有机物为发苂光 DOM, 即 Fluorescent DOM (FDOM), 近年来 FDOM 三维苂光光谱与平行因子 分析相结合被广泛运用于揭示各类天然水体中有色可溶性有机物来源与组成 ${ }^{[17-19]}$.

在我国,已有不少学者开展了湖泊 CDOM 光学特性、来源组成与时空分布方面的研究. 例如, Song 等研 究了我国主要湖泊 CDOM 的光学特性, 发现咸水湖 CDOM 吸收值比淡水湖高 ${ }^{[20]}$; 程庆霖等利用平行因子分 析法 (PARAFAC) 解析了滇池 CDOM 的三维苂光光谱, 显示其外源的贡献率为 $74.18 \%{ }^{[21]}$; Su 等比较了云南 15 个高山湖泊 CDOM 的光学特性, 发现树线以上湖泊 CDOM 的内源性要强于树线以下湖泊 ${ }^{[22]}$; Zhang 等研 究了太湖 CDOM 光学特性的季节变化与空间分布, 发现其时空变化与河流输人和藻类水华程度有关 ${ }^{[23]}$. 抚 仙湖是我国内陆淡水湖中水质较好、蓄水量最大的深水贫营养湖泊,兼具饮用水、防洪、灌溉、渔业及旅游等 综合功能; 尽管我们的前期研究关注了该湖 CDOM 丰度的季节与空间变化及其对水下光辐射衰减的影 响 ${ }^{[2-25]}$, 但仍不清楚其 CDOM 的来源组成, 其时空分布特征亦需进一步研究. 因此, 本研究基于 2017 年 112 月的逐月观测, 结合 CDOM 紫外一可见吸收光谱、三维苂光与平行因子分析法, 探讨了抚仙湖 CDOM 的来 源组成与时空变化特征.

\section{1 材料与方法}

\section{1 抚仙湖概况}

抚仙湖 $\left(24^{\circ} 21^{\prime} \sim 24^{\circ} 38^{\prime} \mathrm{N}, 102^{\circ} 49^{\prime} \sim 102^{\circ} 57^{\prime} \mathrm{E}\right)$ 是我国第二深的淡水湖, 位于云南省玉溪市境内, 流域面 积 $674.69 \mathrm{~km}^{2}$, 湖泊面积 $211 \mathrm{~km}^{2}$, 海拔 $1721 \mathrm{~m}$, 平均水深 $89.6 \mathrm{~m}$, 最大水深 $155 \mathrm{~m}$. 湖区属中亚热带半湿润季 
风气候, 流域年均气温 $15.5^{\circ} \mathrm{C}$, 年降雨量 $800 \sim 1100 \mathrm{~mm}$, 旱雨季分明. $1980 \mathrm{~s}$ 以来, 抗仙湖出现了不同程度的 营养状态上升、透明度下降、浮游植物生物量升高、土著水生植物物种丰度下降等问题 ${ }^{[24,26-29]}$.

\section{2 样品采集与前处理}

在抚仙湖布设 16 个固定采样点 (图 1), 于 2017 年 1-12 进行逐月采样,共采集 192 个水样. 采样过程中, 利用有机玻璃采水器采集水下 $0.5 \mathrm{~m}$ 处水样,随后置于 保温冷藏箱并尽快带回实验室分析. 用高温 $\left(450^{\circ} \mathrm{C}\right.$ 持 续 $4 \mathrm{~h})$ 灼烧过的 Whatman GF/F 膜 $(0.7 \mu \mathrm{m})$ 过滤水 样, 滤液再用 Millipore 膜 $(0.22 \mu \mathrm{m})$ 过滤以用于 CDOM 吸收和三维苂光的测定. 滤液均采用酸洗后的棕色玻 璃瓶装盛, 并保存在 $-20^{\circ} \mathrm{C}$ 冰箱直至测样.

\section{3 参数测定}

1.3.1 CDOM 吸收 水样经 Millipore 膜 $(0.22 \mu \mathrm{m})$ 过滤 后, 滤液采用 Shimadzu UV-2550 紫外一可见分光光度计 测定 $200 \sim 800 \mathrm{~nm}$ 处的吸光度 $\mathrm{OD}(\lambda)$. CDOM 吸收系 数的计算、校正参照公式 (1) 和 (2) ${ }^{[23]}$, 以 $a(254)$ 来表 征 CDOM 丰度 ${ }^{[30-31]}$.

$$
\begin{gathered}
a^{\prime}(\lambda)=2.3030 D(\lambda) / r \\
a(\lambda)=a^{\prime}(\lambda)-a^{\prime}(700) \cdot \lambda / 700
\end{gathered}
$$

式中, $a^{\prime}(\lambda)$ 和 $a(\lambda)$ 分别为未经散射校正的波长为 $\lambda$ 处的吸收系数和经过散射校正过后的波长为 $\lambda$ 处的吸 收系数, $\mathrm{m}^{-1} ; \lambda$ 为波长, $\mathrm{nm} ; r$ 为光程路径, 为 $0.05 \mathrm{~m}$.

吸收光谱斜率 $S$ 值的确定: CDOM 吸收系数随波 长的增加大致呈现指数衰减规律, $S$ 值的计算公 式 $^{[7]}$ 为:

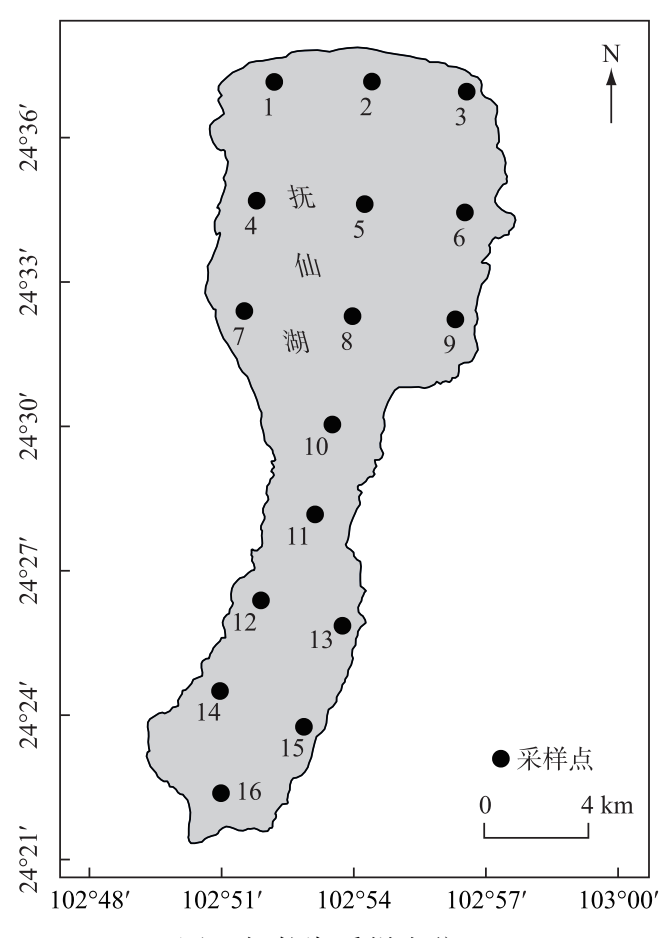

图 1 抚仙湖采样点位置

Fig. 1 Location of sampling sites in Lake Fuxian

$$
a(\lambda)=a\left(\lambda_{0}\right) \exp \left[S\left(\lambda_{0}-\lambda\right)\right]
$$

式中, $\lambda_{0}$ 为参照波长 $(\mathrm{nm})$, 选取 $440 \mathrm{~nm} ; S$ 为指数函数曲线光谱斜率, $\mathrm{nm}^{-1}$. 选取 $275 \sim 295 \mathrm{~nm}$ 波段拟合得到 $S_{275 \sim 295} . S_{275 \sim 295}$ 值越小, 陆源信号越强 ${ }^{[7]}$.

1.3.2 CDOM 三维荧光 水样经 Millipore 膜 $(0.22 \mu \mathrm{m})$ 过滤后, 滤液采用 Hitachi F-7000 三维苂光光谱仪测 定, 得到三维苂光光谱. 激发波长为 $200 \sim 450 \mathrm{~nm}$, 间隔 $5 \mathrm{~nm}$; 发射光波长为 $250 \sim 600 \mathrm{~nm}$, 间隔 $1 \mathrm{~nm}$. 扫描速 度为 $2400 \mathrm{~nm} / \mathrm{min}$, 激发及发射光的狭缝宽度均设置为 $5 \mathrm{~nm}$.

初始数据中包含拉曼散射峰的影响. 把样本的三维苂光图谱扣除 Milli-Q 水的空白三维荧光图谱来去 除纯水散射的影响, 再通过 drEEM 工具箱线性插值的办法剔除第 1 顺序和第 2 顺序瑞利散射峰. 使用 MATLAB R2015b 中的 DOMFluor 工具箱, 对样本三维苂光图谱进行平行因子分析 ${ }^{[32]}$, 得到可识别的荧光峰, 以及 每个样本每个组分的苂光强度和发射、激发光负荷.

苂光指数 $F I$ : 激发波长为 $370 \mathrm{~nm}$ 时, 发射光谱在 450 与 $500 \mathrm{~nm}$ 处的荧光强度比值, 可用于判断微生物 源和陆源 $\mathrm{CDOM}^{[33]}$. 腐殖化指数 $H I X$ : 激发波长为 $255 \mathrm{~nm}$ 时, 发射波长在 $435 \sim 480 \mathrm{~nm}$ 与 $300 \sim 345 \mathrm{~nm}$ 波段 中的菼光强度平均值之比, 用于估计腐殖化程度 ${ }^{[34]}$. 生源指数 $B I X$ : 激发波长为 $310 \mathrm{~nm}$ 时, 发射波长在 380 与 $430 \mathrm{~nm}$ 处苂光强度的比值, 可用于衡量新近的水生生物生产贡献 ${ }^{[34]}$.

1.3.3 Chl.a 浓度 Chl.a 浓度测定方法参照《水和废水监测分析方法》(第四版 $)^{[35]}$.

\section{4 统计分析}

春季为 $3-5$ 月,夏季为 $6-8$ 月,秋季为 $9-11$ 月,冬季为 $1 、 2$ 和 12 月;以 $2 、 5 、 8 、 11$ 月数据开展空间变 化分析, $1^{\#} \sim 9^{\#}$ 点代表 “北部”, $10^{\#} \sim 16^{\#}$ 点代表 “南部”. 使用 IBM SPSS Statistics 25 软件进行统计分析,包括 平均值和标准差计算、平均值 $t$ 检验和 Pearson 相关性分析. 


\section{2 结果}

\section{$2.1 a(254)$ 的时空变化}

抚仙湖 2017 年 12 个月 $a(254)$ 的均值为 $3.47 \pm 0.57 \mathrm{~m}^{-1}$, 范围为 $1.82 \sim 5.22 \mathrm{~m}^{-1}$; 春、夏、秋和冬季的 $a(254)$ 分别为 $3.20 \pm 0.47 、 3.76 \pm 0.64 、 3.67 \pm 0.50$ 和 $3.23 \pm 0.38 \mathrm{~m}^{-1}$, 夏季和秋季的 $a(254)$ 均显著高于冬季和 春季 $(P<0.01) .3$ 月, 全湖各点位的 $a(254)$ 相对较低, 其均值为 $2.63 \pm 0.14 \mathrm{~m}^{-1} ; 8$ 月, 全湖各点位的 $a(254)$ 相对较高, 其均值为 $4.08 \pm 0.4 \mathrm{~m}^{-1}$ (图 2a) $.2 、 5 、 8$ 和 11 月北部 $a(254)$ 的均值分别为 $3.18 \pm 0.20 、 3.68 \pm 0.35$ 、 $3.91 \pm 0.46$ 和 $3.38 \pm 0.84 \mathrm{~m}^{-1}$, 南部 $a(254)$ 的均值分别为 $2.93 \pm 0.42 、 3.24 \pm 0.11 、 4.30 \pm 0.18$ 和 $3.81 \pm 0.51 \mathrm{~m}^{-1}$ (图 3a d), 5 月北部的 $a(254)$ 显著高于南部 $(P<0.01)$.

\section{$2.2 S_{275 ~ 295}$ 的时空变化}

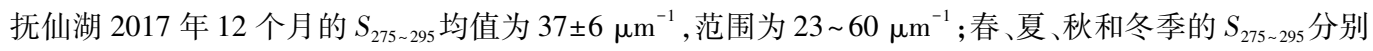
为 $39 \pm 7 、 35 \pm 8 、 35 \pm 4$ 和 $38 \pm 3 \mu \mathrm{m}^{-1}$ ( 图 $2 \mathrm{~b}$ ) , 春季的 $S_{275 ~ 295}$ 显著高于夏季和秋季 $(P<0.05)$, 冬季的 $S_{275 \sim 295}$ 显著 高于秋季 $(P<0.05) .2 、 5 、 8$ 和 11 月北部 $S_{275 \sim 295}$ 的均值分别为 $38 \pm 2 、 35 \pm 2 、 34 \pm 1$ 和 $35 \pm 4 \mu \mathrm{m}^{-1}$, 南部 $S_{275 \sim 295}$ 的 均值分别为 $38 \pm 1 、 39 \pm 2 、 33 \pm 3$ 和 $34 \pm 3 \mu^{-1}$ (图 $3 \mathrm{e} \sim \mathrm{h}$ ), 5 月南部的 $S_{275 \sim 295}$ 显著高于北部 $(P<0.01$ ).
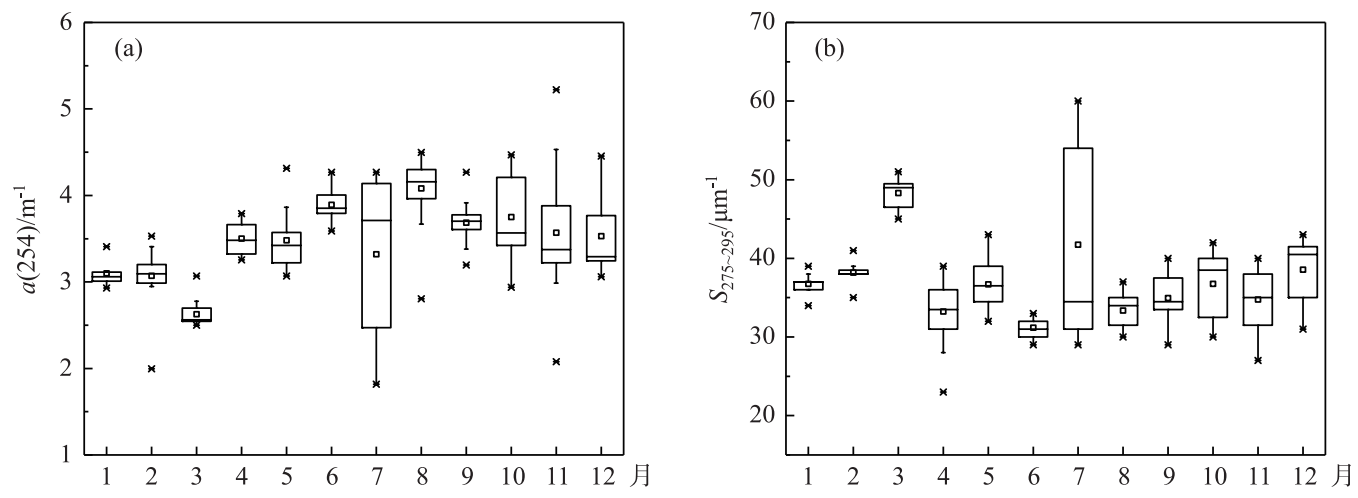

图 2 抚仙湖 $a(254)$ (a) 和 $S_{275 ~ 295}$ (b) 的逐月变化(箱体中间粗线代表中位数,正方形代表均值)

Fig.2 Monthly variations of $a(254)$ (a) and $S_{275 \sim 295}$ (b) of CDOM in Lake Fuxian

( The thick line in the box represents the median value, the square represents the mean value)

\section{$2.3 \mathrm{CDOM}$ 荧光组分苂光特性及时空变化}

经 PARAFAC 分析确定了 4 种苂光组分, 其中 C1 和 C3 代表了类蛋白 (类酪氨酸)、C2 代表了类蛋白 (类色氨酸)、C4 代表了紫外光类腐殖质 (表 1 和图 4);4 种苂光组分苂光强度、Chl.a 浓度间的相关性分析 结果见表 2.12 个月 $\mathrm{C} 1$ 对总苂光强度的平均贡献为 $27.06 \% \pm 27.14 \%, \mathrm{C} 3$ 的平均贡献为 $25.21 \% \pm 11.52 \%, \mathrm{C} 2$ 的平均贡献为 $38.75 \% \pm 20.17 \%, \mathrm{C} 4$ 的平均贡献为 $8.98 \% \pm 4.54 \% .12$ 个月 $F I$ 的均值为 $1.73 \pm 0.14$, 范围为 $1.28 \sim 2.12$ (图 5a) ; 全年 192 个样本的 $H I X$ 值均小于 2,12 个月的均值为 $1.02 \pm 0.37$, 各月份的值差异不大 (图 5b); 12 个月 $B I X$ 的均值为 $1.23 \pm 0.27$, 范围为 $1.03 \sim 2.04$ (图 5c).

表 1 抚仙湖 CDOM 苂光组分特征

Tab.1 Fluorescent component characteristics of CDOM in Lake Fuxian

\begin{tabular}{cccc}
\hline 苂光组分 & $\mathrm{Ex} / \mathrm{Em} / \mathrm{nm}$ & 类型 & 文献中对应组分 $\mathrm{Ex} / \mathrm{Em} / \mathrm{nm}$ \\
\hline $\mathrm{C} 1$ & $225 / 305$ & 类蛋白(类酪氨酸) & $225 \sim 230 / 305 \sim 310^{[36]}$ \\
$\mathrm{C} 2$ & $220 / 340$ & 类蛋白(类色氨酸) & $225 \sim 230 / 340 \sim 350^{[21,36]}$ \\
$\mathrm{C} 3$ & $270 / 300$ & 类蛋白(类酪氨酸) & $275 / 305 \sim 310^{[36]}$ \\
C4 & $250 / 445$ & 类腐殖质 & $230 \sim 260 / 380 \sim 460^{[36-37]}$ \\
\hline
\end{tabular}



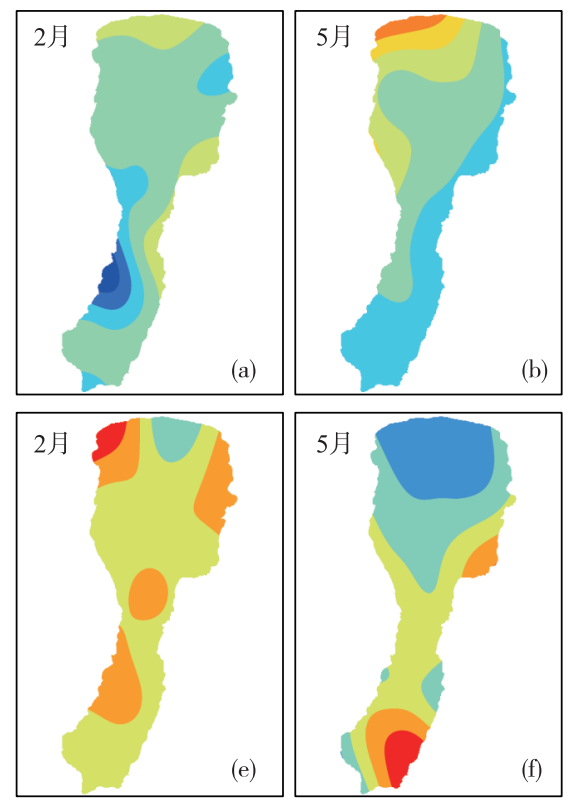
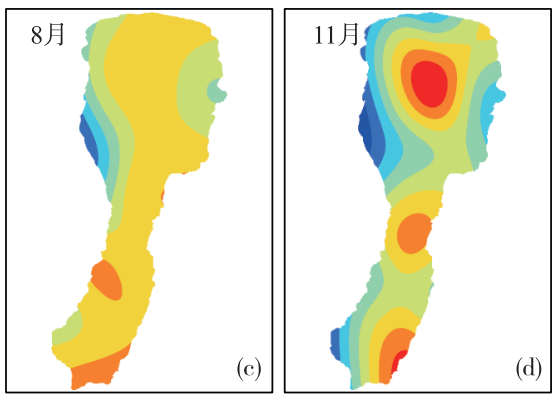

$a(254) / \mathrm{m}^{-1}$
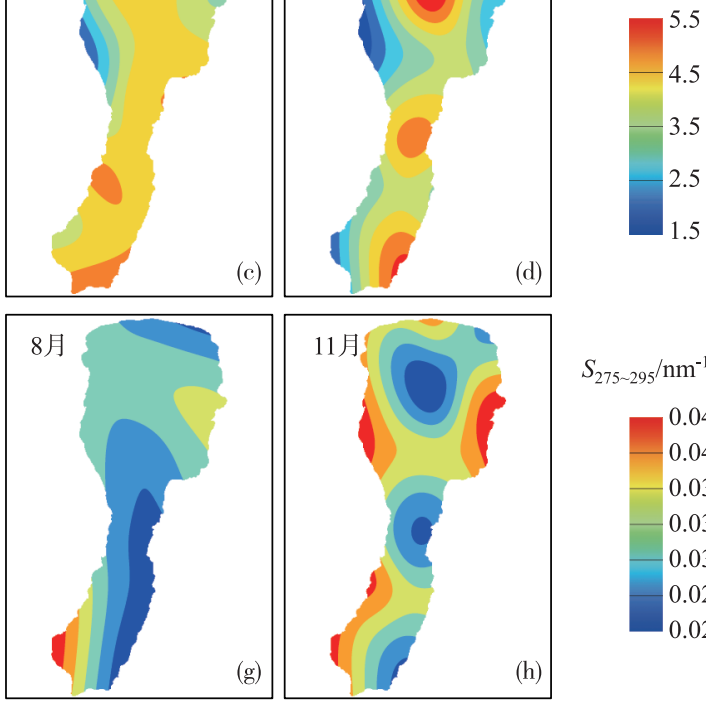

$S_{275 \sim 295} / \mathrm{nm}^{-1}$

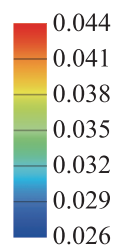

图 32017 年 2 月、 5 月、 8 月、11 月抚仙湖 $a(254)(\mathrm{a} \sim \mathrm{d})$ 和 $S_{275 \sim 295}(\mathrm{e} \sim \mathrm{h})$ 的空间分布

Fig.3 Spatial distributions of $a(254)(\mathrm{a}-\mathrm{d})$ and $S_{275 \sim 295}(\mathrm{e}-\mathrm{h})$ of CDOM

in Lake Fuxian in February, May, August and November of 2017
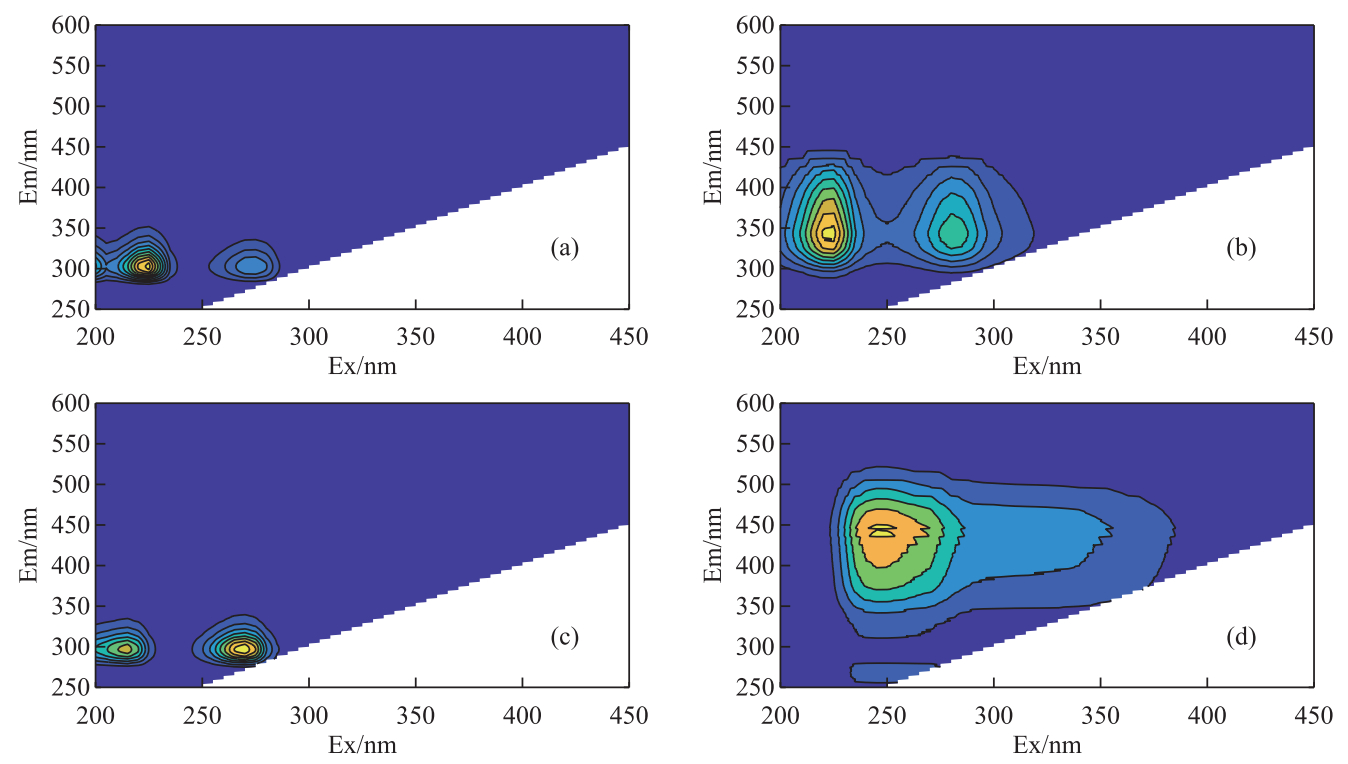

图 4 抚仙湖 4 种菼光组分激发发射苂光谱图 ( $a$ : 组分 $\mathrm{C} 1$; b: 组分 $\mathrm{C} 2$; $\mathrm{c}$ : 组分 $\mathrm{C} 3$; $\mathrm{d}$ : 组分 C4)

Fig.4 Excitation-emission fluorescence spectral shapes of the four components of CDOM in Lake Fuxian (a: Component 1; b: Component 2; c: Component 3; d: Component 4)

根据各组分内外源性质, 可将 $\mathrm{C} 1 \sim \mathrm{C} 4$ 划分为 $\mathrm{C} 1+\mathrm{C} 3$ 和 $\mathrm{C} 2+\mathrm{C} 4$ 两组, 其苂光强度的逐月变化如图 6 所 示. 12 个月 $\mathrm{C} 1+\mathrm{C} 3$ 的苂光强度均值为 $15.68 \pm 22.62$ R.U., 范围为 $2.21 \sim 139.61$ R.U., 月均最小和最大值分别为 

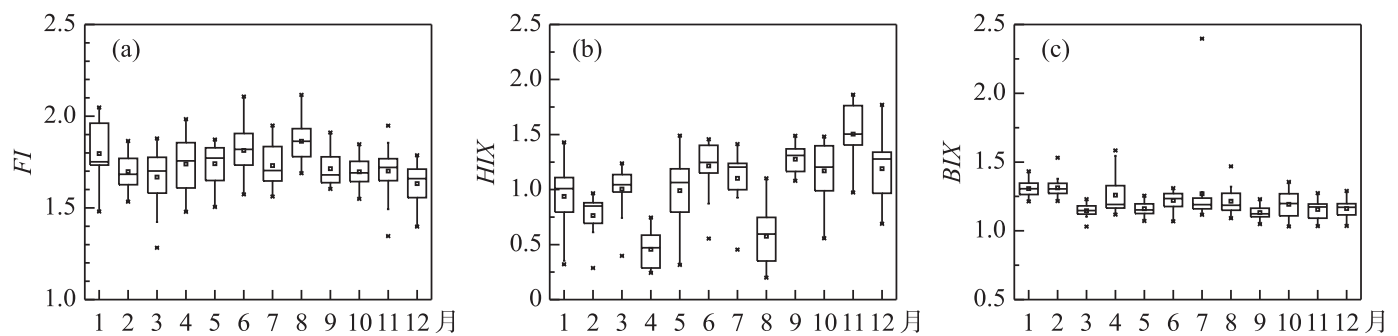

图 5 抚仙湖 CDOM 的 $F I(\mathrm{a}) 、 H I X(\mathrm{~b})$ 和 $B I X(\mathrm{c})$ 的逐月变化 (箱体中间粗线代表中位数,正方形代表均值)

Fig.5 Monthly variations of $F I$ (a), $H I X(\mathrm{~b})$ and $B I X$ (c) of CDOM in Lake Fuxian

(The thick line in the box represents the median value, the square represents the mean value)

表 2 各苂光组分苂光强度、叶绿素 $\mathrm{a}$ 浓度的 Pearson 相关系数矩阵

Tab.2 Pearson correlation between fluorescent intensity of each fluorescent component and chlorophyll-a concentration

\begin{tabular}{rrrrr}
\hline & C1 & C2 & C3 & C4 \\
\hline C2 & 0.109 & & & \\
C3 & 0.126 & $0.230^{* *}$ & & \\
C4 & 0.014 & $0.490^{* *}$ & $0.256^{* *}$ & \\
Chl.a & -0.024 & $0.459^{* *}$ & 0.053 & $0.309^{* *}$ \\
\hline
\end{tabular}

$* P<0.05$, 相关性显著; ${ }^{* *} P<0.01$, 相关性极显著; $n=191$.
$4.36 \pm 1.16$ R.U. ( 11 月) 和 $46.84 \pm 37.10$ R.U. ( 8 月) (图 6a) ; C2+C4 的苂光强度均值为 $4.00 \pm 1.12$ R.U., 范 围为 $2.23 \sim 9.80$ R.U., 月均最小和最大值分别为 $2.60 \pm$ 0.19 R.U. (1 月) 和 $5.64 \pm 0.79$ R.U. ( 8 月) (图 6b). 12 个月 $\mathrm{C} 1+\mathrm{C} 3$ 对总苂光强度的平均贡献为 $65.81 \% \pm$ $15.38 \%, \mathrm{C} 2+\mathrm{C} 4$ 的平均贡献为 $34.19 \% \pm 15.38 \%$.

抚仙湖 2、5、8 和 11 月的苂光组分空间分布如图 7 所示. 2 月, $\mathrm{C} 1+\mathrm{C} 3$ 的苂光强度峰值出现在西北部 近岸和中东部近岸; $\mathrm{C} 2+\mathrm{C} 4$ 无明显的峰值区域. 5 月, $\mathrm{C} 1+\mathrm{C} 3$ 的荧光强度峰值出现在西北部近岸和西部近 岸; $\mathrm{C} 2+\mathrm{C} 4$ 无明显的峰值区域. 8 月, C1 + C 3 的苂光 强度峰值出现在西南部近岸, 东北部近岸和中东部 近岸区域的值也较大; $\mathrm{C} 2+\mathrm{C} 4$ 的荧光强度的峰值出现在西北部近岸和南部近岸, 中部区域的苂光强度值也 稍高. 11 月, $\mathrm{C} 1+\mathrm{C} 3$ 的苂光强度无明显峰值区域; $\mathrm{C} 2+\mathrm{C} 4$ 的峰值区域出现在中北部湖心.
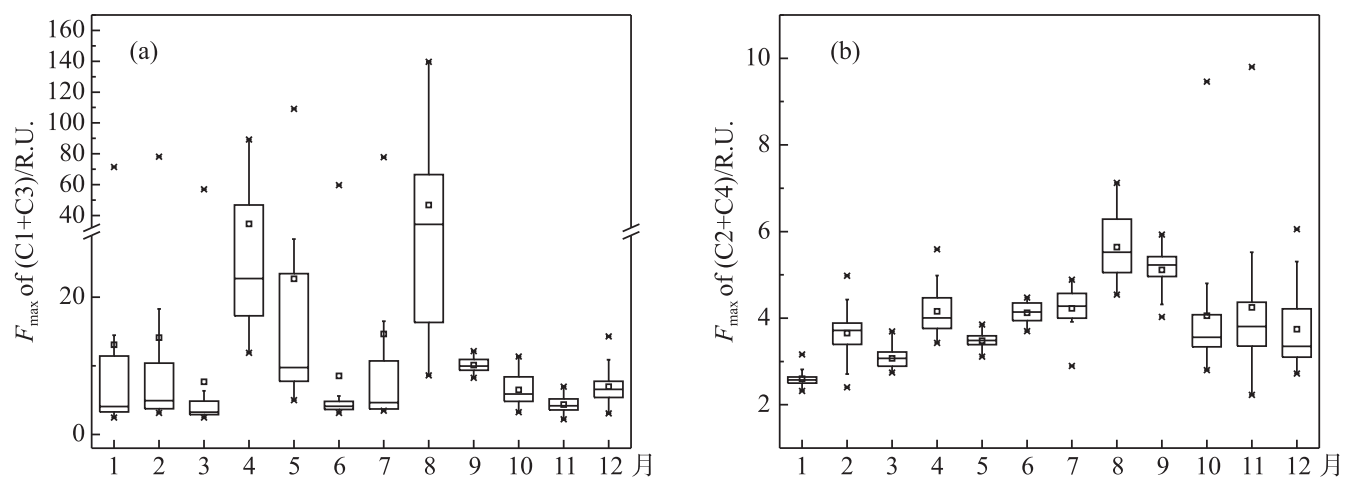

图 6 抚仙湖 $\mathrm{CDOM}$ 的 $\mathrm{C} 1+\mathrm{C} 3(\mathrm{a})$ 和 $\mathrm{C} 2+\mathrm{C} 4(\mathrm{~b})$ 苂光强度的逐月变化 （箱体中间粗线代表中位数,正方形代表均值）

Fig.6 Monthly variations of $\mathrm{C} 1+\mathrm{C} 3$ (a) and $\mathrm{C} 2+\mathrm{C} 4$ (b) of CDOM in Lake Fuxian (The thick line in the box represents the median value, the square represents the mean value)

\section{3 讨论}

CDOM 丰度具有湖区、湖泊间异质性. Song 等对我国 234 个湖泊的调查表明 $a(254)$ 的范围为 1.39 

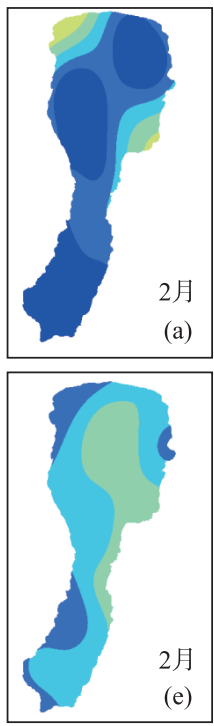
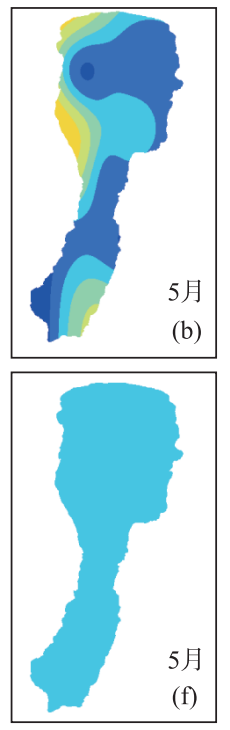

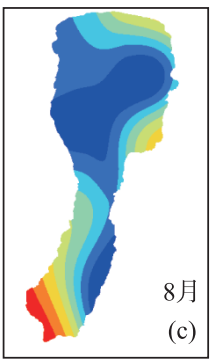

(c)

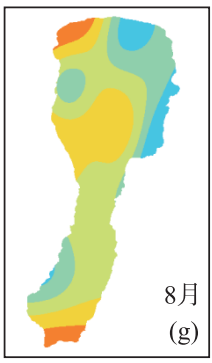

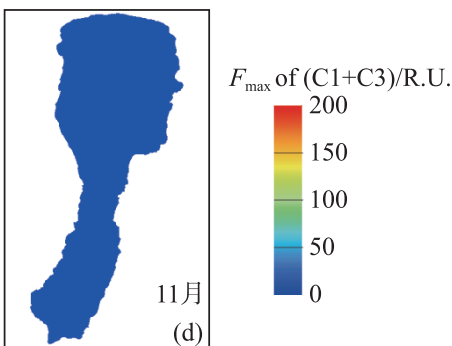

(d)

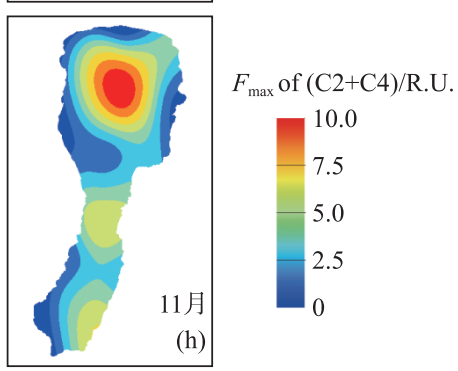

图 7 抚仙湖 2017 年 2、5、8 和 11 月菼光组分 $\mathrm{C} 1+\mathrm{C} 3(\mathrm{a} \sim \mathrm{d})$ 和 $\mathrm{C} 2+\mathrm{C} 4(\mathrm{e} \sim \mathrm{h})$ 的空间分布

Fig.7 Spatial distributions of the fluorescent component $\mathrm{C} 1+\mathrm{C} 3(\mathrm{a}-\mathrm{d})$ and $\mathrm{C} 2+\mathrm{C} 4(\mathrm{e}-\mathrm{h})$ of CDOM in Lake Fuxian in February, May, August and November of 2017

$530.03 \mathrm{~m}^{-1}$, 其中淡水湖泊 $a(254)$ 的平均值为 $19.55 \mathrm{~m}^{-1}$; 在淡水湖泊中, 云贵高原湖区 $a(254)$ 的平均值低 于除青藏高原湖区之外的其他湖区 ${ }^{[20]}$. Zhang 等对我国 22 个湖泊的调查表明 $a(254)$ 的范围为 $1.68 \sim 92.65$ $\mathrm{m}^{-1}$, 平均值为 $13.51 \pm 9.79 \mathrm{~m}^{-1}$, 并指出 $a(254)$ 值与湖泊营养状态有关, $a(254)<4 \mathrm{~m}^{-1}$ 可作为判断贫营养湖 泊的参考依据 ${ }^{[36]}$. 抚仙湖是云贵高原的贫营养湖泊, 其 12 个月 $a(254)$ 的范围为 $1.82 \sim 5.22 \mathrm{~m}^{-1}$, 平均值为 $3.47 \pm 0.57 \mathrm{~m}^{-1}$, 说明该湖 CDOM 丰度处于较低水平.

平行因子分析的结果给出了 4 种组分, 包括类酪氨酸蛋白苂光组分 $(\mathrm{C} 1$ 和 $\mathrm{C} 3$ )、类色氨酸蛋白菼光组分 (C2) 和类腐殖质菼光组分 (C4). 其中, C1 和 C3 可以表征内源 CDOM, 其主要成分包括细菌产生的胞外酶 和生物裂解释放的蛋白质 ${ }^{[37]}$; $\mathrm{C} 2$ 主要为陆源微生物作用而产生的氨基酸类物质, 如来源于垃圾渗滤液和污

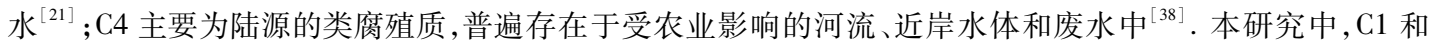
C3 总苂光强度的年均贡献达 77\% (月均贡献范围为 51\% 89\% ), C2 和 C4 的贡献仅为 $23 \%$, 说明抚仙湖 CDOM 以内源组分为主. 抚仙湖是高原清澈型湖泊, 光辐射背景强、漫射衰减系数小 ${ }^{[25]}$, 意味着 CDOM 的光 降解强烈, 而陆源腐殖质类 DOM 比内源蛋白类 DOM 更容易被光降解 ${ }^{[39]}$; 同时, 抚仙湖 CDOM 本底低, 其外 源腐殖质 DOM 能被细菌以及浮游动物利用而转化为内源 $\mathrm{DOM}^{[40]}$, 且外源氮磷营养盐及碳源输人会促使水 体内源蛋白质类 DOM 增加 ${ }^{[41]}$. 类似地, 抚仙湖颗粒态有机碳的研究结果表明, 该湖内源颗粒态有机碳的贡 献 (61\%) 明显大于陆源 (22\%) 和底泥再悬浮 (17\%) 的贡献 ${ }^{[42]}$. 进一步分析发现,抚仙湖 CDOM 苂光特性与 地下水和泉水的相似 ${ }^{[43]}$, 其 $F I 、 H I X$ 和 $B I X$ 特征说明该湖 (内源) CDOM 主要为微生物产生的. 本研究中, $F I$ 相对集中于 1.4 1.9 之间, 说明 CDOM 苂光组分主要为微生物源 ${ }^{[43]}$; BIX 均大于 1 , 与 Huguet 等发现的微生 物主导的内源 CDOM 特性相似 ${ }^{[34]}$; C1、C3 与 Chl. a 相关性不显著, 也说明内源 CDOM 苂光组分主要不是浮 游植物直接产生的; 此外, $H I X$ 较低, 说明腐殖质类 CDOM 主要是生物性残渣产生的且腐殖化程度较低 ${ }^{[44]}$.

抚仙湖 CDOM 丰度为夏、秋季总体高于冬、春季, 与此前针对该湖秋、冬季的研究结果类似 ${ }^{[25]}$. 夏、秋季 相对高温促使水体中微生物活性增加, 且夏、秋季正值云南高原的雨季, 降水充沛不仅会将陆源 CDOM 直接 带人湖体 ${ }^{[8-9]}$,亦可为水体微生物异养代谢提供相对充足的碳源; 光化学降解是水体 DOM 损失的重要途径 之一 ${ }^{[7]}$, 云南高原夏、秋季的总辐射低于冬、春季 ${ }^{[45]}$, 加之抚仙湖紫外线 B 穿透深度的季节异质性 (如, 秋季 小于冬季 ${ }^{[25]}$, 或意味着该湖夏、秋季 CDOM 的光降解弱于冬、春季. 根据抚仙湖海口站观测资料 (2010- 
2014 年), $2 、 5 、 8$ 和 11 月的平均降水量分别为 $9.28 、 94.08 、 104.86$ 和 $18.18 \mathrm{~mm}$. CDOM 丰度在旱季 ( 2 和 11 月) 并无南北部间的显著性差异, 可能与降水稀少导致外源有机污染物输人少有关; 5 月, $S_{275 \sim 295}$ 的南北差异 显示北部的陆源 CDOM 高于南部, 而北部人湖河流的径流贡献较大 (澄江县境内人湖河流的年径流总量占 流域的 $63.7 \%)^{[46]}$ 且有机污染高于南部 ${ }^{[47]}$, 加之 5 月降水量大, 进而导致湖体北部的 $a(254)$ 显著高于南部; 8 月, 南部部分近岸区的 CDOM 丰度较高 (内源和外源 CDOM 的苂光强度均较高), 或与大鲫鱼沟等南部主 要人湖河流的贡献有关. 类似地, 抗仙湖内外源 CDOM 的空间分布具有一定的季节异质性,或受流域土地利 用类型、入湖河流分布和降雨条件等的综合影响. 例如, CDOM 内源苂光组分 $(\mathrm{C} 1+\mathrm{C} 3)$ 的苂光强度在 2 月的 北部近岸、5月的西北部和西部近岸较高,这些区域存在较多的农业、生活污染源或来自旅游业的污染(西岸 旅游业较发达且 5 月为旅游旺季), Dai 等的研究亦显示抚仙湖北部和西部人湖河流的有机污染总体较南部 和东部高 ${ }^{[47]}$, 而有机污染物输人会促使对应区域近岸水体微生物生长 ${ }^{[48]}$; CDOM 外源苂光组分 $(\mathrm{C} 2+\mathrm{C} 4)$ 的 苂光强度在 8 月的西北部和南部近岸较高, 或与这些区域的外源 CDOM 易受雨水冲刷分别进人山冲河 (西 北部)、大鲫鱼沟 (南部) 等人湖河流进而输人近岸水体有关. 需指出的是, 11 月 $\mathrm{C} 2+\mathrm{C} 4$ 的苂光强度峰值出现 在湖心敞水区, 与一般湖库陆源 CDOM 分布规律相反 ${ }^{[19]}$, 需进一步研究. 当然, CDOM 的空间分布情况, 亦 不排除水动力条件的影响.

虽然目前抚仙湖的 CDOM 丰度较低且以微生物内源为主, 但该湖已呈现营养状态上升、浮游植物生物 量增加的趋势 ${ }^{[26-27]}$, 意味着藻源 CDOM 有进一步增加的可能; 随着社会经济的迅速发展, 抚仙湖流域内点 源、面源污染逐渐加剧, 或可导致外源 CDOM 输人增加, 亦可能间接促进内源 CDOM 增加. 需指出的是, 监测 数据表明抚仙湖已呈现有机污染水平升高的趋势 ${ }^{[26-27]}$, 并可引起该湖水体透明度下降 ${ }^{[24,28]}$, 加之 CDOM 是 该湖光衰减的主要影响因子之一 ${ }^{[24-25]}$, 意味着光穿透深度下降与 CDOM 光化学降解作用减弱 (丰度升高) 可 能存在正反馈作用, 进而不利于沉水植物的生长与分布. 根据本研究结果, 抚仙湖 CDOM 丰度较高的区域主 要分布于人口相对集中区域或靠近受污染河流人湖口, 故应加强 (尤其雨季) 对主要人湖河流 (有机) 污染 的管控. 鉴于抗仙湖是典型的清澈型贫营养深水湖泊, 今后可开展 CDOM 垂向剖面分析, 亦有必要结合光衰 减、热力分层及有关其他水质参数乃至 CDOM 的流域输人等开展进一步研究, 深人探讨抚仙湖 CDOM 的内 外源贡献及其迁移转化规律与驱动机制.

\section{4 结论}

抚仙湖 CDOM 丰度较低, 2017 年 12 个月的 CDOM 均以内源为主. 利用平行因子分析法得到了 4 种荧 光组分,包括 2 种类酪氨酸蛋白苂光组分、 1 种类色氨酸蛋白苂光组分和 1 种类腐殖质荧光组分; CDOM 表 现出较强的微生物源荧光特性, 腐殖化特征不明显. 时空变化方面, 冬、春季 CDOM 丰度较低, 夏、秋季较高; CDOM 丰度及内外源组分的空间分布具有季节异质性, 内源 CDOM 主要分布在受人类活动影响较大的近岸 区,可能与流域土地利用、河流输人、降雨、温度、光辐射等因素有关.

致谢: 王溪市抚仙湖管理局综合行政执法二大队协助了野外调查, 李光旭、王荣华、黄立成、王玮璐、秦江、刘 宁超等协助了野外调查或样品分析,特此致谢!

\section{5 参考文献}

[ 1 ] Zhang Y, Zhang E, Yin Y et al. Characteristics and sources of chromophoric dissolved organic matter in lakes of the Yungui Plateau, China, differing in trophic state and altitude. Limnology and Oceanography, 2010, 55 (6) : 2645-2659. DOI: $10.4319 / \mathrm{lo} .2010 .55 .6 .2645$.

[ 2 ] Yao X, Zhang Y, Zhu G et al. Resolving the variability of CDOM fluorescence to differentiate the sources and fate of DOM in Lake Taihu and its tributaries. Chemosphere, 2011, 82(2) : 145-155. DOI: 10.1016/j.chemosphere.2010.10.049.

[ 3 ] Coble AA, Marcarelli AM, Kane ES. Ammonium and glucose amendments stimulate dissolved organic matter mineralization in a Lake Superior tributary. Journal of Great Lakes Research, 2015, 41(3) : 801-807. DOI: 10.1016/j.jglr.2015.05.015.

[ 4 ] Shang P, Lu Y, Du Y et al. Climatic and watershed controls of dissolved organic matter variation in streams across a gradient of agricultural land use. Science of the Total Environment, 2018, 612: 1442-1453. DOI: 10.1016/j. scitotenv.2017. 08.322 . 
[ 5 ] Cole JJ, Prairie YT, Caraco NF et al. Plumbing the global carbon cycle: Integrating inland waters into the terrestrial carbon budget. Ecosystems, 2007, 10(1) : 171-184. DOI: 10.1007/s.

[ 6 ] Tranvik LJ, Downing JA, Cotner JB et al. Lakes and reservoirs as regulators of carbon cycling and climate. Limnology and Oceanography, 2009, 54(6) : 2298-2314. DOI: 10.4319/lo.2009.54.6_part_2.2298.

[ 7 ] Helms JR, Stubbins A, Ritchie JD et al. Absorption spectral slopes and slope ratios as indicators of molecular weight, source, and photobleaching of chromophoric dissolved organic matter. Limnology and Oceanography, 2008, 53(3) : 955969. DOI: $10.4319 /$ lo.2008.53.3.0955.

[ 8 ] Zhang Y, Gao G, Shi K et al. Absorption and fluorescence characteristics of rainwater CDOM and contribution to Lake Taihu, China. Atmospheric Environment, 2014, 98: 483-491. DOI: 10.1016/j.atmosenv.2014.09.038.

[ 9 ] Zhou Y, Zhang Y, Shi K et al. Dynamics of chromophoric dissolved organic matter influenced by hydrological conditions in a large, shallow, and eutrophic lake in China. Environmental Science and Pollution Research, 2015, 22 (17): 1299213003. DOI: $10.1007 / \mathrm{s} 11356-015-4556-\mathrm{x}$.

[10] Lu YH, Bauer JE, Canuel EA et al. Effects of land use on sources and ages of inorganic and organic carbon in temperate headwater streams. Biogeochemistry, 2014, 119(1/2/3) : 275-292. DOI: 10.1007/s10533-014-9965-2.

[11] Prairie YT. Carbocentric limnology: Looking back, looking forward. Canadian Journal of Fisheries and Aquatic Sciences, 2008, 65(3) : 543-548. DOI: 10.1139/f08-011.

[12] Lapierre JF, Guillemette F, Berggren M et al. Increases in terrestrially derived carbon stimulate organic carbon processing and $\mathrm{CO}_{2}$ emissions in boreal aquatic ecosystems. Nature Communications, 2013, 4: 7. DOI: 10.1038/ncomms3972.

[13] Seekell D, Lapierre JF, Ask J. The influence of dissolved organic carbon on primary production in northern lakes. Limnology and Oceanography, 2015, 60(4) : 1276-1285. DOI: 10.1002/lno.10096.

[14] Thrane JE, Hessen DO, Andersen T. The absorption of li ght in lakes: Negative impact of dissolved organic carbon on primary productivity. Ecosystems, 2014, 17(6) : 1040-1052. DOI: 10.1007/s10021-014-9776-2.

[15] Zhou Y, Jeppesen E, Zhang Y et al. Dissolved organic matter fluorescence at wavelength 275/342 $\mathrm{nm}$ as a key indicator for detection of point-source contamination in a large Chinese drinking water lake. Chemosphere, 2016, 144: 503-509. DOI: $10.1016 /$ j.chemosphere.2015.09.027.

[16] Yamashita Y, Jaffe R, Maie N et al. Assessing the dynamics of dissolved organic matter (DOM) in coastal environments by excitation emission matrix fluorescence and parallel factor analysis (EEM-PARAFAC). Limnology and Oceanography, 2008, 53(5) : 1900-1908. DOI: 10.4319/lo.2008.53.5.1900.

[17] Zhao Y, Song K, Wen Z et al. Seasonal characterization of CDOM for lakes in semiarid regions of Northeast China using excitation-emission matrix fluorescence and parallel factor analysis (EEM-PARAFAC). Biogeosciences, 2016, 13 ( 5 ): 1635-1645. DOI: 10.5194/bg-13-1635-2016.

[18] Margolin AR, Gonnelli M, Hansell DA et al. Black Sea dissolved organic matter dynamics: Insights from optical analyses. Limnology and Oceanography, 2018, 63(3) : 1425-1443. DOI: 10.1002/lno.10791.

[19] Zhou Y, Yao X, Zhang Y et al. Response of dissolved organic matter optical properties to net inflow runoff in a large fluvial plain lake and the connecting channels. Science of the Total Environment, 2018, 639: 876-887. DOI: 10.1016/j.scitotenv. 2018.05.180.

[20] Song K, Shang Y, Wen Z et al. Characterization of CDOM in saline and freshwater lakes across China using spectroscopic analysis. Water Research, 2019, 150: 403-417. DOI: 10.1016/j.watres.2018.12.004.

[21] Cheng QL, Zheng BH, Wang SR et al. Optical signatures of chromophoric dissolved organic matter in water body of Tien Lake. Spectroscopy and Spectral Analysis, 2014, 34(3): 698-703. [程庆霖, 郑丙辉, 王圣瑞等. 滇池水体有色溶解性 有机质 (CDOM) 三维菼光光谱特征. 光谱学与光谱分析, 2014, 34(3): 698-703.]

[22] Su Y, Chen F, Liu Z. Comparison of optical properties of chromophoric dissolved organic matter ( CDOM) in alpine lakes above or below the tree line: Insights into sources of CDOM. Photochemical \& Photobiological Sciences, 2015, 14(5): 1047-1062. DOI: 10.1039/c4pp00478g.

[23] Zhang Y, Yin Y, Liu X et al. Spatial-seasonal dynamics of chromophoric dissolved organic matter in Lake Taihu, a large eutrophic, shallow lake in China. Organic Geochemistry, 2011, 42 (5) : 510-519. DOI: 10.1016/j. orggeochem.2011. 03.007 .

[24] Zhou Q, Zhang Y, Li K et al. Seasonal and spatial distributions of euphotic zone and long-term variations in water transpar- 
ency in a clear oligotrophic Lake Fuxian, China. Journal of Environmental Sciences, 2018, 72: 185-197. DOI: 10.1016/j. jes.2018.01.005.

[25] Zhou QC, Zhang YL, Zhou YQ et al. Spectral attenuation of ultraviolet and visible radiation and its relationship with chromophoric dissolved organic matter in autumn/winter in Lake Fuxian, China. J Lake Sci, 2016, 28(6) : 1316-1327. DOI: 10.18307/2016.0617. [ 周起超, 张运林, 周永强等. 抚仙湖秋、冬季光衰减特征及其与有色可溶性有机物的关系. 湖泊科学, 2016, 28(6): 1316-1327.]

[26] Gao W, Chen Y, Xu M et al. Trend and driving factors of water quality change in Lake Fuxian (1980-2011). J Lake Sci, 2013, 25(5): 635-642. DOI: 10.18307/2013.0503. [高伟, 陈岩, 徐敏等. 抚仙湖水质变化(1980-2011 年) 趋势与 驱动力分析. 湖泊科学, 2013, 25(5): 635-642.]

[27] Chen JX, Lv Y, Zhao ZF et al. Using the multidimensional synthesis methods with non-parameter test, multiple time scales analysis to assess water quality trend and its characteristics over the past 25 years in the Fuxian Lake, China. Science of the Total Environment, 2019, 655: 242-254. DOI: 10.1016/j.scitotenv.2018.11.144.

[28] Zhou Q, Wang W, Huang L et al. Spatial and temporal variability in water transparency in Yunnan Plateau lakes, China. Aquatic Sciences, 2019, 81(2) : 36. DOI: 10.1007/s00027-019-0632-5.

[29] Wang S, Wang J, Li M et al. Six decades of changes in vascular hydrophyte and fish species in three plateau lakes in Yunnan, China. Biodiversity and Conservation, 2013, 22(13) : 3197-3221. DOI: 10.1007/s10531-013-0579-0.

[30] Henderson RK, Baker A, Murphy KR et al. Fluorescence as a potential monitoring tool for recycled water systems: A review. Water Research, 2009, 43(4) : 863-81. DOI: 10.1016/j.watres.2008.11.027.

[31] Ou HS, Wei CH, Deng Y et al. Principal component analysis to assess the composition and fate of impurities in a large river-embedded reservoir: Qingcaosha Reservoir. Environmental Science Processes \& Impacts, 2013, 15( 8) : 1613-21. DOI: $10.1039 / \mathrm{c} 3 \mathrm{em} 00154 \mathrm{~g}$.

[32] Stedmon C, Bro R. Characterizing dissolved organic matter fluorescence with parallel factor analysis: A tutorial. Limnology and Oceanography-Methods, 2008, 6: 572-579. DOI: 10.4319/lom.2008.6.572.

[33] McKnight DM, Boyer EW, Westerhoff PK et al. Spectrofluorometric characterization of dissolved organic matter for indication of precursor organic material and aromaticity. Limnology and Oceanography, 2001, 46(1) : 38-48. DOI: 10.4319/lo. 2001.46.1.0038.

[34] Huguet A, Vacher L, Relexans S et al. Properties of fluorescent dissolved organic matter in the Gironde Estuary. Organic Geochemistry, 2009, 40(6) : 706-719. DOI: 10.1016/j.orggeochem.2009.03.002.

[35] Editorial board of “Water and wastewater monitoring and analysis method”, Ministry of Environmental Protection of the People's Republic of China ed. Monitoring and analysis methods of water and wastewater: fourth edition. Beijing: China Environmental Science Press, 2002. [ 国家环境保护总局《水和废水监测分析方法》编委会. 水和废水监测分析方 法: 第 4 版. 北京: 中国环境科学出版社, 2002.]

[36] Zhang Y, Zhou Y, Shi K et al. Optical properties and composition changes in chromophoric dissolved organic matter along trophic gradients: Implications for monitoring and assessing lake eutrophication. Water Research, 2018, 131: 255-263. DOI: $10.1016 /$ j.watres.2017.12.051.

[37] Huang CC, Li Y, Wang Q et al. Components optical property of CDOM in lake Taihu based on three-dimensional excitation emission matrix fluorescence. J Lake Sci, 2010, 22 (3) : 375-382. DOI: 10.18307/2010.0309. [黄昌春, 李云梅, 王桥 等. 基于三维荧光和平行因子分析法的太湖水体 CDOM 组分光学特征. 湖泊科学, 2010, 22(3): 375-382.]

[38] Osburn CL, Handsel LT, Mikan MP et al. Fluorescence tracking of dissolved and particulate organic matter quality in a river-dominated estuary. Environmental Science \& Technology, 2012, 46(16) : 8628-8636. DOI: 10.1021/es3007723.

[39] Zhu WZ, Zhang J, Yang GP. Mixing behavior and photobleaching of chromophoric dissolved organic matter in the Changjiang River estuary and the adjacent East China Sea. Estuarine, Coastal and Shelf Science, 2018, 207: 422-434. DOI: $10.1016 /$ j.ecss.2017.07.019.

[40] Sun H, Zhang YD, Yu JL et al. Contribution of allochthonous dissolved organic carbon to the carbon source of planktonic crustaceans in Lake Fuxian. J Lake Sci, 2017, 29(4) : 887-895. DOI: 10.18307/2017.0412. [孙欢, 张永东, 于谨否 等. 外源溶解性有机碳对抚仙湖甲壳类浮游动物碳源的贡献. 湖泊科学, 2017, 29(4): 887-895.]

[41] Brandão LPM, Brighenti LS, Staehr PA et al. Distinctive effects of allochthonous and autochthonous organic matter on CDOM spectra in a tropical lake. Biogeosciences, 2018, 15(9) : 2931-2943. DOI: 10.5194/bg-15-2931-2018. 
[42] Chen J, Yang H, Zeng Y et al. Combined use of radiocarbon and stable carbon isotope to constrain the sources and cycling of particulate organic carbon in a large freshwater lake, China. Science of the Total Environment, 2018, 625: 27-38. DOI: 10.1016/j.scitotenv.2017.12.275.

[43] Birdwell JE, Engel AS. Characterization of dissolved organic matter in cave and spring waters using UV-Vis absorbance and fluorescence spectroscopy. Organic Geochemistry, 2010, 41(3) : 270-280. DOI: 10.1016/j.orggeochem.2009.11.002.

[44] Ohno T, Chorover J, Omoike A et al. Molecular weight and humification index as predictors of adsorption for plant- and manure-derived dissolved organic matter to goethite. European Journal of Soil Science, 2007, 58(1) : 125-132. DOI: 10. 1111/j.1365-2389.2006.00817.x.

[45] Liu L, Liu JD, Wu DR et al. Distributed simulation of global solar radiation over mountainous region in Yunnan Province. Meteorological and Environmental Sciences, 2014, 37(1): 13-19. [刘玲, 刘建栋, 邬定荣等. 云南山地太阳总辐射的 分布式模拟. 气象与环境科学, $2014,37(1): 13-19$.

[46] Xia TX, Pan JZ, Liu XH et al. Non-point source pollution characteristics in Fuxianhu Lake watershed and variation law of $\mathrm{N}$ and P in lake water. Journal of Agro-Environment Science, 2008, 27(4) : 1340-1345. [夏天翔, 潘继征, 刘雪华等. 抚仙湖水体 N P 变化及其非点源污染特征. 农业环境科学学报, 2008, 27(4) : 1340-1345.]

[47] Dai X, Zhou Y, Ma W et al. Influence of spatial variation in land-use patterns and topography on water quality of the rivers inflowing to Fuxian Lake, a large deep lake in the plateau of southwestern China. Ecological Engineering, 2017, 99: 417428. DOI: 10.1016/j.ecoleng.2016.11.011.

[48] Aburto-Medina A, Shahsavari E, Salzman SA et al. Elucidation of the microbial diversity in rivers in south-west Victoria, Australia impacted by rural agricultural contamination (dairy farming). Ecotoxicology and Environmental Safety, 2019, 172 : 356-363. DOI: 10.1016/j.ecoenv.2019.01.112. 